\title{
Eletroquímica de solos modais e de sua matéria orgânica em ambientes tropicais ${ }^{1}$
}

\author{
Marihus Altoé Baldotto², Ary Carlos Xavier Velloso ${ }^{3}$ \\ http://dx.doi.org/10.1590/0034-737X201461060018
}

\begin{abstract}
RESUMO
A química do solo vem estudando o comportamento da fração mineral dos solos tropicais intemperizados, incluindo a eletroquímica, o desenvolvimento de cargas e o fenômeno de adsorção. Por causa do amplo espectro a ser coberto no entendimento do comportamento da fração mineral, somado às dificuldades metodológicas, o estudo da complexa fração orgânica dos solos foi relativamente menos desenvolvido. A atual proposta revisita os primeiros trabalhos referentes à química de solos tropicais, buscando relacioná-los com dados de eletroquímica dos estoques de carbono do solo. O objetivo deste trabalho foi revisar aspectos de eletroquímica de solos, determinar e relacionar os pontos de carga zero, por diferentes métodos, os potenciais da dupla camada elétrica a eletroquímica do húmus de solos modais brasileiros, em uma sequência típica de intemperismo, visando a gerar informações para o seu manejo e conservação. Os métodos de estimativa dos ponto de carga zero dos solos apresentam resultados variados, mas com a mesma tendência entre os solos. Há predomínio de cargas negativas em ambas as camadas dos solos estudados. As cargas negativas dos coloides estão diretamente associadas à disponibilidade de elétrons do húmus e ambas diminuem com o estádio de intemperismo do solo.
\end{abstract}

Palavras-chave: química do solo, matéria orgânica, troca iônica, ponto de carga zero.

\section{ABSTRACT}

\section{Electrochemistry of modal soils and its organic matter in tropical environments}

Soil chemistry soils studies the behavior of the mineral fraction of weathered tropical soils, including electrochemistry, development of charges and adsorption phenomenon. Because of the broad spectrum to be covered in the understanding of the behavior of mineral fraction added to the methodological difficulties, the study of complex soil organic fraction was relatively less developed. The current proposal revisits the early works related to the chemistry of tropical soils with the objective to relate them with electrochemical data of soil carbon stocks. The objective of this study was to review aspects of soil electrochemistry, to determine and to relate the points of zero charge by different methods, the potentials of the electric double layer and the electrochemistry of humus of Brazilian modal soils in a typical sequence of weathering, aiming to generate information for their management and conservation. The methods for estimating the point of zero charge of the soils show different results, but with the same trend among soils. There is a predominance of negative charges on both layers of soils. The negative charges of the colloids are significantly associated with the availability of electrons from the humus and both decrease with the weathering stage of the soil.

Key words: electrochemistry, ionic exchange, organic matter,point of zero charge, soil chemistry.

\footnotetext{
Recebido para publicação em 10/06/2013 e aprovado em 24/06/2014.

${ }^{1}$ Trabalho financiado pela FAPERJ (E-26/110.633/2007) e pela FAPEMIG (APQ-02395-10).

2 Engenheiro-Agrônomo, Químico. Doutor. Instituto de Ciências Agrárias, Universidade Federal de Viçosa, Campus de Florestal, Rodovia LMG 818, Km 06, 35690-000, Florestal, Minas Gerais, Brasil.marihus@ufv.br (autor para correspondência).

${ }^{3}$ Engenheiro-Agrônomo. Doutor. Professor Titular Aposentado da Universidade Federal Rural do Rio de Janeiro e da Universidade Estadual do Norte Fluminense "Darcy Ribeiro". velloso@uenf.br
} 


\section{INTRODUÇÃO}

A química de superfície de solos tropicais vem sendo estudada, ao longo das últimas décadas (Stern, 1924; Schofield, 1949; Parks \& Bruyn, 1962; Parks, 1965; Raij \& Peech, 1972; Raij, 1973a,b; Uehara, 1979; Uehara \& Gilman, 1980 a, b; Sposito, 1981; Leal et al., 1983; Magalhães \& Page, 1984a,b; Sposito, 1989; Siqueira et al., 1990a, b, c; Chaves \& Trajano, 1992; Stumm, 1992; Oliveira et al., 1993; Manzato et al., 1994; Silva et al., 1996; Peixoto, 1997; Sposito, 1998; Fontes et al., 2001; Struyk \& Sposito, 2001; Baldotto, 2006; Sposito, 2008; Baldotto et al., 2010). A carga líquida total das partículas dos solos $\left(\sigma_{p}\right)$ pode ser positiva, zero ou negativa, segundo a equação (01): $\sigma_{\mathrm{p}}=$ $\sigma_{0}+\sigma_{\mathrm{H}}+\sigma_{\text {is }}+\sigma_{\text {os }}$ (Sposito, 2008). Nessa equação, as variáveis $\sigma_{0}, \sigma_{\mathrm{H},} \sigma_{\text {is }}$ e $\sigma_{\text {os }}$ representam as cargas permanente, variável, advindas de complexos de superfícies por adsorção específica ("inner sphere") e não específica ("outer sphere"), respectivamente, as quais serão descritas a seguir:

A $\sigma_{0}$ é a carga permanente, resultante de substituição isomórfica, $\sigma_{H}$ é a carga variável, e desenvolve-se na fração húmus do solo, nos “óxidos" e nas argilas silicatadas $1: 1$ (Sposito, 2008); $\sigma_{\text {is }}$ e $\sigma_{\text {os }}$ são as cargas de complexos inner sphere e referem-se à carga líquida total de íons (que não sejam $\mathrm{H}^{+}$e $\mathrm{OH}^{-}$) (Sposito, 2008). Diferentemente da carga intrínseca, $\sigma_{\text {is }}$ e $\sigma_{\text {os }}$ resultam somente de constituintes da solução do solo, adsorvidos nas superfícies das partículas (Leal \& Velloso, 1973; Charlet \& Sposito, 1987).

$\mathrm{Na}$ equação $(01), \sigma_{\mathrm{p}}$ precisa ser balanceada, no caso de diferir de zero, por outro tipo de carga que resulte de íons da solução do solo que não estejam ligados a complexos de superfície, mas sejam adsorvidos pelas partículas do solo, isto é, íons da camada difusa $\left(\sigma_{\mathrm{D}}\right)$ ou dupla camada elétrica (Stern, 1924; Sposito, 2008). Esses íons da camada difusa movem-se livremente, na solução do solo, ao mesmo tempo permanecendo nas proximidades das superfícies das partículas para criarem uma carga de superfície efetiva, contrabalançando $\sigma_{\mathrm{p}}$ (Sposito, 2008).

A soma algébrica de $\sigma_{0}$ e $\sigma_{\mathrm{H}}$ representa a carga superficial intrínseca do solo. Essas cargas resultam, principalmente, dos componentes estruturais dos adsorventes, por exemplo, dos constituintes iônicos dos minerais e dos grupamentos protonáveis, nos sólidos orgânicos e inorgânicos. A contribuição relativa de cada uma dessas cargas dependerá do estádio de intemperismo e do teor e qualidade da matéria orgânica do solo (Leal et al., 1983; Oliveira et al., 1993; Manzato et al., 1994; Peixoto, 1997; Fontes et al., 2001; Sposito, 2008; Baldotto et al., 2010).
Com relação à mineralogia do solo, desde que a fonte principal de carga permanente esteja na cavidade "siloxane" (Sposito, 2008), $\sigma_{\text {o }}$ predomina nos solos nos estádios inicial e intermediário de intemperismo (Jackson \& Sherman, 1953). Por outro lado, os solos em estádio de intemperismo avançado são enriquecidos com argilominerais com grupamentos anfóteros (silanols e aluminols), além de grupos funcionais da matéria orgânica (ácidos carboxílicos e fenólicos) e superfícies hidroxiladas, nos óxidos de Fe e de Al. Nestes últimos, de carga variável, $\sigma_{\mathrm{H}}$ domina $\sigma_{\mathrm{o}}$ e as variações de seu $\mathrm{pH}$ influenciam fortemente o desenvolvimento de cargas (Baldotto et al., 2010). Nesses casos, cada radical funciona como ácido fraco, gerando cargas de superfície negativas ou positivas, quando o pH está acima ou abaixo de seu potencial de dissociação (pKa), respectivamente (Sposito, 2008).

Para a avaliação global dos constituintes do solo, o valor do Ponto de Carga Zero (PCZ) indica o pH no qual o balanço de cargas dos coloides do solo é nulo, isto é, $\sigma_{\mathrm{p}}=0$. Nesse valor de $\mathrm{pH}$ não existe carga líquida relativa aos íons adsorvidos na camada difusa (Sposito, 2008), ou seja, $\sigma_{\mathrm{P}}+\sigma_{\mathrm{D}}=0$. Essa condição pode ser experimentalmente estabelecida, verificando-se, num determinado campo elétrico, o valor de $\mathrm{pH}$ no qual as partículas deixam de se mover (medida da mobilidade em eletroforese) ou, então, o valor de pH no qual as partículas sedimentam ("medida da floculação"). Esse ponto de carga zero assinala a ausência de íons adsorvidos, movendo-se livremente na camada difusa e, também, representa a intensificação das forças entre as partículas responsáveis pelos feitos de coagulação. O PCZ desempenha, portanto, papel importante na formação de agregados e na retenção de íons adsorvidos, contra os efeitos da lixiviação, especialmente nos solos de carga variável (Sposito, 2008).

O ponto de carga líquida zero (PZNC - Point of Zero Net Charge) pode ser definido como o $\mathrm{pH}$ no qual a carga líquida dos íons adsorvidos (outros que não $\mathrm{H}^{+} \mathrm{e} \mathrm{OH}^{-}$) zera. Se $\mathrm{q}_{+}$e $\mathrm{q}$, na equação (02), a seguir $\left(\mathrm{q}_{+}-\mathrm{q}_{-}=\sigma_{\text {is }}+\right.$ $\left.\sigma_{\text {os }}+\sigma_{\mathrm{D}}=0\right)$, representam cargas de cátion e ânion adsorvidos, respectivamente, então $\mathrm{q}_{+}=\mathrm{q}$. no PZNC. $\mathrm{O}$ valor do PZNC dependerá da escolha do íon índice (“index íon"), a ser trabalhado para determinar-se o potencial, sendo comumente utilizados os produtos da solubilização de $\mathrm{KCl}$ e de $\mathrm{NaCl}$. O valor de PZNC é pouco afetado quando se utiliza um "eletrólito indiferente". Então, no PZNC, $\sigma_{\text {is }}$ não contribui, se o sistema estiver saturado com o eletrólito indiferente (Sposito, 2008). Portanto, íons móveis na camada difusa existem no PZNC, mas não no PZC, pois, por definição, neutralizam as demais cargas $\sigma_{0}, \sigma_{\mathrm{H}} \sigma_{\text {is }}$ e $\sigma_{\text {os, }}$, como visto na equação (01).

Rev. Ceres, Viçosa, v. 61, n.6, p. 1012-1021, nov/dez, 2014 
A formação de complexos com cátions na superfície aumenta o PCZ, enquanto a formação de complexos com ânions decresce o PCZ (Leal \& Velloso, 1973; Chartlet \& Sposito, 1987; Sposito, 2008). Esses deslocamentos do PCZ não requerem adsorção específica, mas somente complexação na superfície. O deslocamento do PCZ, após adsorção adicional de um íon, não pode, portanto, ser interpretado como evidência de adsorção específica. Esse deslocamento apenas evidencia que complexos foram formados na superfície, em obediência à lei do balanço de carga (Sposito, 2008).

$\mathrm{O}$ ponto de carca protônica líquida zero (PZNPC Point of Zero Net Proton Charge) é o $\mathrm{pH}$ no qual $\sigma_{\mathrm{H}}=0$ (Sposito, 2008). Como propriedade geral, $\sigma_{\mathrm{H}}$ decresce (fica negativo) com o aumento do $\mathrm{pH}$ (i.e., $\Delta_{\mathrm{\sigma H}} / \Delta_{\mathrm{pH}}$ é sempre negativo). Essa propriedade independe da composição ou da força iônica da solução e, também, da natureza das partículas do solo (inorgânicas ou orgânicas). O PZNPC e as cargas superficiais do solo de carga variável $\left(\sigma_{H}\right)$ são, frequentemente, determinados pelo método de titulação potenciométrica, que se baseia numa série de titulações de suspensões de terra com íons determinantes de potencial $\left(\mathrm{H}^{+}\right.$ou $\left.\mathrm{OH}^{-}\right)$, numa determinada concentração de "eletrólito indiferente" (Sposito, 2008).

O PZNPC corresponde ao ponto de interseção da curva $\sigma_{\mathrm{H}} v s . \mathrm{pH}$, naquela concentração de eletrólitos. Quando várias concentrações de eletrólitos indiferentes são usadas, o ponto de interseção das várias curvas $\sigma_{\mathrm{H}}$ vs. $\mathrm{pH}$ representa o ponto de efeito salino nulo (PESN), isto é, o ponto de carga variável $\left(\sigma_{H}\right)$ não é afetado pela variação da concentração do eletrólito $\left(\Delta_{\mathrm{I}}\right.$ $=$ variação força iônica $)$, ou seja, $\sigma_{\mathrm{H} / \Delta \mathrm{I}}=0($ Sposito, 2008).

O PCZ é um ponto de referência para se avaliar o sinal da carga líquida da superfície, no $\mathrm{pH}$ atual do solo, ou seja, se ela está carregada positivamente ( $\mathrm{pH}<\mathrm{PCZ})$, ou negativamente ( $\mathrm{pH}>\mathrm{PCZ})$. Com o PCZ é possível determinar o potencial da dupla camada elétrica $\left(\Psi_{0}\right.$, $\mathrm{mV}$ ), pela equação (03), que é a equação simplificada de Nernst $\left(\Psi_{0}=0,059(\mathrm{PCZ}-\mathrm{pH})\right.$ descrita por Uehara \& Gillman (1980), em que o PCZ pode ser estimado pelo PESN e o pH é medido em água.

Os valores de PCZ e de $\Psi_{\mathrm{O}}$ têm diversas aplicações importantes, como as previsões sobre a tendência à floculação e à dispersão dos coloides do solo, o grau de intemperismo e a formação de complexos de superfície (Sposito, 2008).

Um método relativamente simples, para se estimar se a carga líquida dos coloides do solo é negativa, zero ou positiva, consiste em analisar os valores de $\mathrm{pH}$ determinados em KCl 1 mol/L e em água (Uehara \& Gillman, $1980 \mathrm{a}, \mathrm{b})$. A estimativa é dada por $\Delta \mathrm{pH}$, que é igual à diferença entre $\mathrm{pH}_{\mathrm{KCl}}$ e $\mathrm{pH}_{\mathrm{H} 2 \mathrm{O}}$ (Equação 04) $\mathrm{O} \Delta \mathrm{pH}$ tem sido utilizado na caracterização de solos tropicais. Valor negativo ou positivo de $\Delta \mathrm{pH}$ é indicativo de predominância de cargas líquidas negativas ou positivas nos coloides, respectivamente. De acordo com Uehara \& Gilman (1980a), uma estimativa do PCZ pode ser feita indiretamente pela equação (05): $\mathrm{PCZ}=2 \mathrm{pH}_{\mathrm{KCl}}-\mathrm{pH}_{\mathrm{H} 2 \mathrm{O}}$ em que os valores de $\mathrm{pH}_{\mathrm{KCl}}$ e de $\mathrm{pH}_{\text {Н2о }}$ provém da determinação do $\mathrm{pH}$ em $\mathrm{KCl} 1 \mathrm{~mol} \mathrm{~L}^{-1}$ e em água, ambos na relação solo: extrator igual a 1: 2,5.

Baldotto et al. (2010) estudaram a eletroquímica das substâncias húmicas, medindo sua capacidade de transferir elétrons, indicando a relação entre estas cargas negativas e o PCZ. Para planejamento do aumento dos teores de carbono no solo (estabilização, sequestro, etc), é fundamental conhecer a relação entre a densidade de carga e seu sinal. Assim, são necessários estudos que relacionem propriedades termodinâmicas da matéria orgânica com a química de superfície dos solos, objetivando o entendimento dos mecanismos de interação entre os compartimentos orgânico e mineral e de estabilização do húmus (Stevenson, 1994; Piccolo, 2001; Struyk \& Sposito, 2001; Sutton \& Sposito, 2005), nos ecossistemas tropicais (Baldotto et al., 2010).

O objetivo deste trabalho foi revisar aspectos de eletroquímica de solos, determinar e relacionar os pontos de carga zero por diferentes métodos, os potenciais da dupla camada elétrica e a eletroquímica do húmus de solos modais brasileiros em uma sequência típica de intemperismo, visando a gerar informações para o seu manejo e conservação.

\section{MATERIAL E MÉTODOS}

\section{Amostras}

Foram amostradas as camadas superficial $(0$ a $20 \mathrm{~cm})$ e subsuperficial $(20$ a $40 \mathrm{~cm}$ ) de solos modais descritos durante a I Reunião de Classificação e Correlação de Solos (Embrapa, 1980), em diferentes estádios de intemperismo, classificados como (Embrapa, 2013): Chernossolo Argilúvico Órtico vértico, Chernossolo Rêndzico Saprolítico típico, Luvissolo Crômico Pálico abrúptico, Argissolo Vermelho-Amarelo Distrófico e Latossolo Amarelo Coeso típico (Tabela 1).

\section{Atributos dos solos e da matéria orgânica}

Os atributos dos solos (Tabela 2) e da matéria orgânica (Tabela 3), determinados e detalhadamente descritos em Baldotto et al. (2010), foram reunidos neste trabalho para relacioná-los aos pontos de carga zero e aos potenciais da dupla camada elétrica, determinados como descrito a seguir. 
Tabela 1. Classificação, vegetação, coordenadas e localização dos solos

\begin{tabular}{|c|c|c|c|c|c|}
\hline \multirow{2}{*}{$\begin{array}{l}\text { Amostra } \\
\mathbf{N}^{\mathbf{0}}\end{array}$} & \multicolumn{2}{|c|}{ Classificação ${ }^{(1)}$} & & \multicolumn{2}{|c|}{ Coordenadas } \\
\hline & USDA & Vegetação $^{(2)}$ SiBCS & & Latitude & $\overline{\text { Longitude }}$ \\
\hline 1 & Typic Calciustoll & Chernossolo Rêndzico Saprolítico típico & Pastagem & $21^{\circ} 25^{\prime} \mathrm{S}$ & $41^{\circ} 41^{\prime} \mathrm{O}$ \\
\hline 2 & Vertic Argiustoll & Chernossolo Argilúvico Órtico vértico & Pastagem & $21^{\circ} 13^{\prime} \mathrm{S}$ & $41^{\circ} 49^{\prime} \mathrm{O}$ \\
\hline 3 & Typic Kanhaplustult & Luvissolo Cromico Pálico abrúptico & Pastagem & $21^{\circ} 12^{\prime} \mathrm{S}$ & $41^{\circ} 50^{\prime} \mathrm{O}$ \\
\hline 4 & Ultic Paleustalf & Argissolo Vermelho- Amarelo distrófico & Pastagem & $21^{\circ} 13^{\prime} \mathrm{S}$ & $41^{\circ} 50^{\prime} \mathrm{O}$ \\
\hline 5 & Typic Haplustox & Latossolo Amarelo Coeso típico & Pastagem & $21^{\circ} 43^{\prime} \mathrm{S}$ & $41^{\circ} 20^{\prime} \mathrm{O}$ \\
\hline
\end{tabular}

(1) Classificação: USDA = Soil Survey Staff (2006) e Sistema Brasileiro de Classificação de Solos (Embrapa, 2013); Predominantemente com braquiárias.

\section{Determinação do $\Delta p H$ e predição do ponto de carga zero}

A determinação do $\Delta \mathrm{pH}$ foi obtida pela equação (04). As determinações do $\mathrm{pH}$ em água e em $\mathrm{KCl}$ foram realizadas, preparando-se suspensões de solo e água destilada e de solo e solução de $\mathrm{KCl} 1 \mathrm{~mol} \mathrm{~L}^{-1}$, na relação solo: água ou solo:solução igual a 1:2,5, obtida pela adição de $25 \mathrm{~mL}$ de água ou de solução a $10 \mathrm{~cm}^{3}$ de solo, seguida de agitação para completa homogeneização e formação da suspensão. Após 30 a 60 minutos, foi determinado o pH das suspensões. De posse dos dados de $\mathrm{pH}$ em $\mathrm{KCl}$ e em $\mathrm{H}_{2} \mathrm{O}$, foi realizada a predição do PCZ pela equação (05).

\section{Determinação experimental do ponto de carga zero}

Para este trabalho, os pontos de carga zero estimados experimentalmente foram obtidos pelos métodos do ponto de efeito salino nulo (PESN) e do ponto de carga protônica líquida zero (PCPLZ), conforme Sposito (2008).

Para determinação do PESN, subamostras de solo de $10 \mathrm{~cm}^{3}$ foram agitadas, por cinco minutos, em agitador horizontal, com $25 \mathrm{~mL}$ de água deionizada ou 1,00 mol $\mathrm{L}^{-1}$ de $\mathrm{KCl}$. Depois de um tempo de equilíbrio de $30 \mathrm{mi}-$ nutos, as amostras foram suspensas, agitando-se com bastão de vidro, e o pH foi medido, usando-se um eletrodo previamente calibrado. $\mathrm{O}$ valor de $\Delta \mathrm{pH}$ foi obtido pela diferença entre o $\mathrm{pH}$ em $\mathrm{KCl}$ e o $\mathrm{pH}$ em $\mathrm{H}_{2} \mathrm{O}$. Sendo conhecidos os valores de $\Delta \mathrm{pH}$ para a construção do espaço experimental, assumiu-se que $\Delta \mathrm{pH}<0, \Delta \mathrm{pH}=0$, $\Delta \mathrm{pH}>0$ indicaram solos eletronegativos, no ponto de carga, e eletropositivos, respectivamente. Para cada solo, 18 amostras de $5 \mathrm{~g}$ foram divididas em três grupos de seis amostras, que receberam, em erlenmeyers, $25 \mathrm{~mL}$ de $\mathrm{NaCl} 0,1 \mathrm{~mol} \mathrm{~L}^{-1}$, $\mathrm{NaCl} 0,01 \mathrm{~mol} \mathrm{~L}^{-1}$ e $\mathrm{NaCl} 0,001$ mol L ${ }^{-1}$, respectivamente. As amostras de cada um desses grupos de concentração salina receberam 0,$0 ; 0,1$; 0,2; 0,3; 0,4 e 0,5 mL de $\mathrm{HCl} \mathrm{0,4} \mathrm{mol} \mathrm{L}^{-1}$, para os solos eletronegativos; 0,3 ; 0,4 e $0,5 \mathrm{~mL}$ de $\mathrm{HCl} \mathrm{0,4} \mathrm{mol} \mathrm{L}^{-1} \mathrm{e}$ 0,$1 ; 0,2$ e $0,3 \mathrm{~mL}$ de $\mathrm{NaOH} 0,4 \mathrm{~mol} \mathrm{~L}^{-1}$, para os solos próximos ao ponto de carga e 0,$0 ; 0,1 ; 0,2 ; 0,3 ; 0,4$ e 0,5 $\mathrm{mL}$ de $\mathrm{NaOH} 0,4 \mathrm{~mol} \mathrm{~L}^{-1}$, para os solos eletropositivos.
Em seguida, os frascos foram agitados por 5 minutos, em agitador horizontal, e deixados em repouso, por 24 horas. Ao final deste período, sem agitar os frascos, determinou-se o $\mathrm{pH}$ do sobrenadante. As curvas de titulação foram obtidas, relacionando-se, em um gráfico, os valores de $\mathrm{pH}$ determinados nos seis pontos de cada concentração do eletrólito $(\mathrm{NaCl})$, em função da adsorção de $\mathrm{OH}^{-}$ou $\mathrm{H}^{+}$, obtida de acordo com as quantidades de ácido/base $(\mathrm{HCl} / \mathrm{NaOH})$ adicionadas, expressa em $\mathrm{cmol}_{\mathrm{c}} /$ $\mathrm{kg}$. O ponto de cruzamento das três curvas foi definido como o valor de $\mathrm{pH}$ equivalente ao PESN do solo em questão.

Os PCPLZ das amostras foram determinados de forma análoga, porém relacionando ao $\mathrm{pH}$ apenas as adsorções de $\mathrm{H}^{+}$ou de $\mathrm{OH}^{-}$na concentração de $0,01 \mathrm{~mol}$ $\mathrm{L}^{-1}$ de $\mathrm{NaCl}$. Ao invés do ponto de interseção das curvas, em diferentes concentrações, como no PESN, o PCPLZ foi obtido no ponto no qual a curva que relacionou a variável independente, adsorção de $\mathrm{H}^{+}(\mathrm{y})$, corta o eixo da variável independente $\mathrm{pH}$ (x) (Sposito, 2008).

\section{Potencial da dupla camada elétrica}

A partir da estimativa experimental do PCZ pelo método do PESN, foi determinado o potencial da dupla camada elétrica $\left(\Psi_{\mathrm{O}}, \mathrm{mV}\right)$, pela equação $(03)$, empregando-se o valor de $\mathrm{pH}$ medido em água.

\section{Análises estatísticas}

A análise dos dados fundamentou-se na estatística descritiva, por se tratar de amostras aleatórias simples (fatores aleatórios). Estimou-se a correlação linear de Pearson entre as variáveis estimadas para a eletroquímica do solo e da matéria orgânica (Steel \& Torrie, 1980). Foi, também, realizado o teste de agrupamento pela variância mínima com distância euclidiana, usando-se a análise multivariada dos dados (Cruz, 2006).

\section{RESULTADOS E DISCUSSÃO}

Os resultados das estimativas dos pontos de carga zero e dos potenciais da dupla camada elétrica foram diferentes entre os solos modais (Embrapa, 2013) amostrados (Tabela 4). 


\begin{tabular}{|c|c|c|c|c|c|c|c|c|c|c|}
\hline \multirow{3}{*}{$\begin{array}{l}\text { Amostra } \\
\text { Classe }\end{array}$} & \multicolumn{10}{|c|}{ Atributos $^{(1)}$} \\
\hline & $\mathbf{C}$ & $\mathbf{A F}$ & $\mathbf{A H}$ & \multirow[t]{2}{*}{$\mathbf{H}$} & AH/AF & EC & \multirow[t]{2}{*}{$\mathbf{K i}$} & Areia & Silte & Argila \\
\hline & & $\mathrm{g} \mathrm{kg}^{-1}$ & & & & Mg ha & & \multicolumn{3}{|c|}{$\mathrm{g} \mathrm{kg}^{-1}$} \\
\hline Chernossolo Rêndzico & 22,6 & 1,21 & 3,44 & 19,2 & 2,9 & 47,9 & 2,6 & 400 & 180 & 420 \\
\hline Chernossolo Argilúvico & 17,2 & 2,42 & 3,66 & 10,8 & 1,5 & 35,8 & 1,9 & 300 & 230 & 470 \\
\hline Luvissolo Crômico & 16,2 & 3,33 & 3,11 & 6,6 & 1,0 & 48,6 & 1,8 & 400 & 330 & 270 \\
\hline ArgissoloVermelho- Amarelo & 13,6 & 3,32 & 1,90 & 9,3 & 0,6 & 27,5 & 1,8 & 600 & 160 & 240 \\
\hline Latossolo Amarelo & 13,1 & 2,14 & 0,83 & 8,9 & 0,4 & 35,6 & 1,6 & 600 & 20 & 380 \\
\hline
\end{tabular}

(1) Atributos: $\mathrm{C}, \mathrm{AF}, \mathrm{AH} \mathrm{e} \mathrm{H}=$ carbono total, ácidos fúlvicos, ácidos húmicos e huminas, respectivamente; $\mathrm{EC}=$ Estoque de carbono $(0-0,20 \mathrm{~m}) ; \mathrm{Ki}^{2}$ relação molar $\mathrm{SiO} / \mathrm{Al} \mathrm{O}_{3} ; \mathrm{Areia}$, silte e argila = frações granulométricas.

\section{Amostra}

Classe

Atributos $^{(1)}$

\begin{tabular}{|c|c|c|c|c|c|c|c|c|c|c|c|c|c|}
\hline \multirow{2}{*}{ Classe } & \multirow[t]{2}{*}{$\mathbf{p H}$} & \multirow{2}{*}{\multicolumn{2}{|c|}{$\mathrm{mg} \mathrm{dm}^{-3}$}} & \multirow{2}{*}{\multicolumn{8}{|c|}{$\mathrm{cmol}_{\mathrm{c}} \mathrm{kg}^{-1}$}} & \multirow{2}{*}{\multicolumn{2}{|c|}{$\%$}} \\
\hline & & & & & & & & & & & & & \\
\hline Chernossolo Rêndzico & 7,0 & 10,2 & 0,12 & 43,3 & 0,4 & 0,14 & 0,0 & 0,8 & 44,0 & 44,0 & 44,8 & 98 & 0 \\
\hline Chernossolo Argilúvico & 7,2 & 7,3 & 0,21 & 18,2 & 0,4 & 0,10 & 0,0 & 2,8 & 18,9 & 18,9 & 21,7 & 87 & 0 \\
\hline Luvissolo Crômico & 6,6 & 7,5 & 0,10 & 9,9 & 0,2 & 0,06 & 0,0 & 2,4 & 10,3 & 10,3 & 12,7 & 81 & 0 \\
\hline Argissolo Vermelho -Amarelo & 6,4 & 5,4 & 0,15 & 4,7 & 2,6 & 0,09 & 0,0 & 3,3 & 7,5 & 7,5 & 10,8 & 70 & 0 \\
\hline Latossolo Amarelo & 5,5 & 5,3 & 0,19 & 1,8 & 1,9 & 0,09 & 0,1 & 4,6 & 4,0 & 4,1 & 8,6 & 46 & 2 \\
\hline
\end{tabular}

${ }^{(1)}$ Atributos: $\mathrm{pH}$ obtido em relação solo: água igual a 1:2,5; Pe K $=$ Extrator Mehlich- $1 ; \mathrm{Ca}^{2+}, \mathrm{Mg}^{2+} \mathrm{e} \mathrm{Al}{ }^{3+}=$ Extrator $\mathrm{KCl} 1 \mathrm{~mol} \mathrm{~L}^{-1} ; \mathrm{H}+\mathrm{Al}=\mathrm{Extrator} \mathrm{CaOAc} 0,5 \mathrm{~mol} \mathrm{~L}{ }^{-1}$ a pH 7,0; $\mathrm{SB}=\operatorname{soma}$ de bases; t e T $=$ capacidade de troca de cátions efetiva e potencial $(\mathrm{pH} 7,0)$, respectivamente; $\mathrm{V}=$ saturação por bases; $\mathrm{m}=$ saturação por $\mathrm{Al}^{3+}$ 


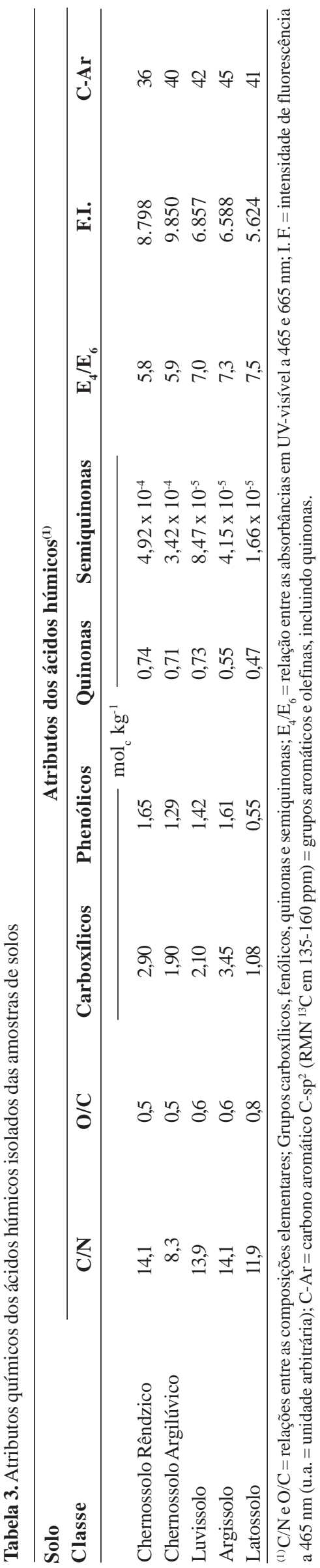

A Figura 1 é um exemplo das titulações para determinação do PESN. O gráfico mostra que, nos pontos que receberam ácido $(\mathrm{HCl})$, o $\mathrm{pH}$ aumentou na medida em que a concentração da solução salina $(\mathrm{NaCl})$ diminuiu. Isso pode ser explicado pelo efeito salino, segundo o qual os íons $\mathrm{H}^{+}$se expressam menos, num ambiente com elevada concentração de sais (menor atividade). Somase a isto a provável atividade biológica mais baixa e, portanto, menor interferência na atividade de $\mathrm{H}^{+}$(Sposito, 2008).

Os valores de PCZ, estimados experimentalmente ou calculados (Equação 05), não foram concordantes (Tabela 4). Essas discrepâncias ocorreram, possivelmente, em função de a concentração de $\mathrm{KCl} 1 \mathrm{~mol} \mathrm{~L}^{-1}$ ser muito elevada. Por exemplo, $25 \mathrm{~mL}$ de $\mathrm{KCl} 1 \mathrm{~mol} \mathrm{~L}^{-1}$, adicionados à $10 \mathrm{~g}$ de solo, levam à concentração de $250 \mathrm{cmol}_{c}$ $\mathrm{kg}^{-1}$ de $\mathrm{K}^{+}$. Essa concentração dobra, se padronizada a um teor de argila de 50\%. Por exemplo, para um solo com $500 \mathrm{~g} \mathrm{~kg}^{-1}$ de argila, ocorreria adsorção de $500 \mathrm{cmol}_{\mathrm{c}}$ $\mathrm{kg}^{-1}$ de $\mathrm{K}^{+}$. Além disso, as argilas predominantemente encontradas em solos em estádio avançado de intemperismo apresentam, geralmente, CTC entre 10 a $25 \mathrm{cmol}_{\mathrm{c}}$ $\mathrm{kg}^{-1}$, dezenas de vezes menor do que aquela magnitude de concentração adicionada a partir do $\mathrm{KCl} 1 \mathrm{~mol} / \mathrm{L}^{-1}$. Assim, o estudo de concentrações mais baixas de $\mathrm{KCl}$ ou a alteração do fator 2 da fórmula devem ser buscados, no sentido de melhorar a capacidade preditiva do método, visando à avaliação do PCZ do solo com maior exatidão. Outro fator que poderia explicar a diferença entre os valores de PCZ calculado e medido é a complexidade do sistema. Se somente o $\mathrm{KCl}$ estivesse presente em solução, sendo um eletrólito indiferente, poderia haver maior aproximação dos PCZ calculado e experimental. Entretanto, outros íons estão presentes nos solos, sujeitos, por exemplo, à adsorção específica. Se forem ânions, esse fato leva à diminuição do valor do PCZ e, consequentemente, gera incrementos de carga negativa na superfície do coloide.

Segundo Raij \& Peech (1972), de forma geral, a presença de óxidos de ferro e de alumínio tendem a aumentar o PCZ, enquanto a presença de minerais de argila silicatada, com cargas negativas estruturais ou permanentes, tende a diminuir o PCZ. A presença de matéria orgânica, especialmente em solos tropicais muito imtemperizados, tende a reduzir o PCZ, pois seus grupos carboxílicos se dissociam em valores de $\mathrm{pH}$ mais baixos que o dos óxidos de ferro e de alumínio. Assim, o PCZ reflete a composição mineralógica e o conteúdo de matéria orgânica de um solo.

A Figura 2 mostra o dendrograma, obtido pela análise multivariada de todos os dados apresentados nas Tabelas 1, 2, 3 e 4, no qual os solos aparecem agrupados de acordo com a sua verossimilhança, determinada pela 


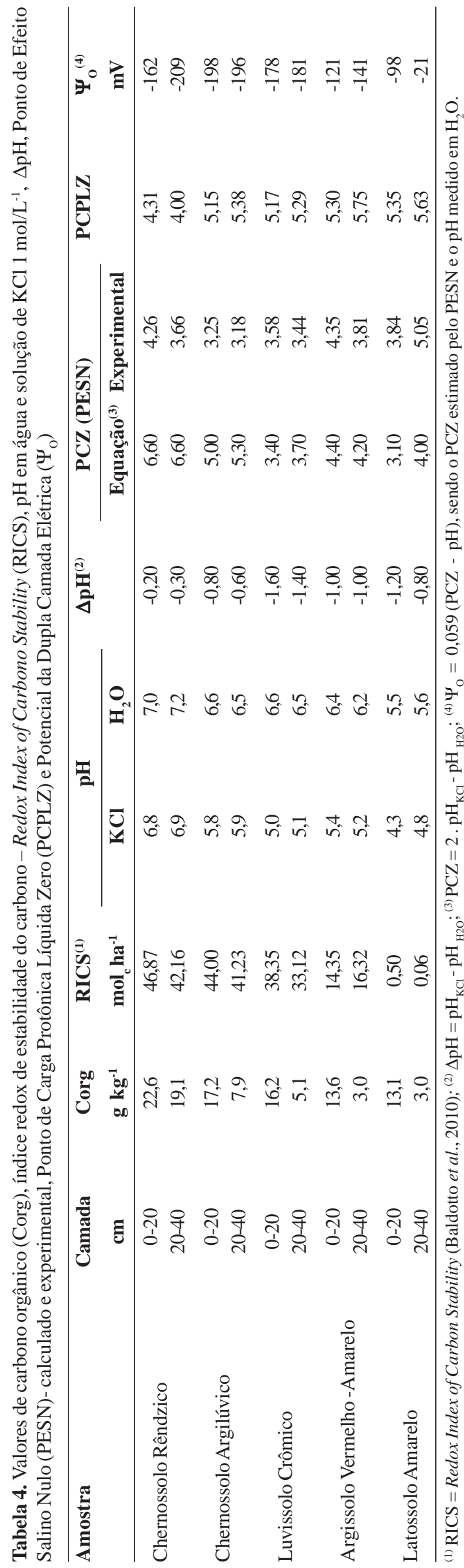

distância Euclidiana (Cruz, 2006). Observa-se, no dendrograma, que os solos em estádio menos avançado de intemperismo (Chernossolos e Luvissolos) aparecem num mesmo grupo, separados dos solos em estádio avançado de intemperismo (Argissolo e Latossolo), os quais foram agrupados juntos. Assim, o dendrograma, considerando-se todo o conjunto de dados, converge para as diferenças observadas nos pontos de carga zero e nos potenciais da dupla camada elétrica, estimados neste estudo, bem como sua ordenação em função dos atributos do solo. Essas correlações (Tabela 5) possibilitam a obtenção de indicadores de manejo de solos, em ecossistemas naturais e agrários, a partir das propriedades eletroquímicas. Baldotto et al. (2010) apresentaram uma discussão detalhada desses atributos, evidenciando a relação entre a gênese, a química, a fertilidade do solo e a química e a eletroquímica do húmus extraído. Neste trabalho, a eletroquímica dos solos é estudada como um todo (frações mineral e orgânica), por meio dos pontos de carga zero e dos potenciais da dupla camada elétrica. Os resultados, portanto, confirmam, também, que a química de superfície do solo é um significativo indicador de gênese, intemperismo, formação e manejo de solos tropicais.

Todos os solos estudados apresentaram maior potencial da dupla camada elétrica na camada 0 a $20 \mathrm{~cm}$. Os aumentos dos estoques e da composição de grupos funcionais doadores de carga da matéria orgânica dos solos (que foi muito diferente entre os solos modais) foram acompanhados por diminuição dos valores de PCZ e por mais negativos $\Psi_{0}$ (Tabela 5). Esse efeito indica que a preservação e o aumento dos estoques de carbono são primeiro passo para a recuperação das propriedades dos Latossolos (aumentos de CTC e da disponibilidade de nutrientes, simultaneamente à diminuições da adsorção específica de $\mathrm{P}$ e da atividade tóxica do $\mathrm{Al}^{3+}$ ).

Além das correlações já mencionadas, a Tabela 5 revela que os atributos fundamentais para o estudo de solos (físicos e químicos) estão intimamente relacionados com os fenômenos biológicos. Especialmente, a química das substâncias húmicas é também um reflexo da qualidade ecológica dos sistemas estudados, tendo tanto a estabilização dos seus estoques quanto sua capacidade de gerar carga sido beneficiadas pela elevada disponibilidade de bases, baixa acidez e alta densidade de carga negativa dos solos menos intemperizados, em relação aos altamente intemperizados, pobres em nutrientes, de baixa CTC e ácidos (Baldotto et al., 2010).

Contudo, os resultados também mostraram que nos solos mais intemperizados, a despeito de sua maior necessidade de incrementos da quantidade e da qualidade da matéria orgânica, para melhorar sua fertilidade, ocor-

Rev. Ceres, Viçosa, v. 61, n.6, p. 1012-1021, nov/dez, 2014 
rem os menores estoques de carbono, com a menor disponibilidade de cargas negativas. Infere-se, assim, que produção e a preservação das formas de carbono é maior nos solos menos intemperizados, de maiores área superficial específica, capacidade de troca iônica (argilas de maior atividade), disponibilidade de bases, além de menores adsorção de $\mathrm{P}$ e acidez. Nos solos muito intemperizados, como o Argissolo e o Latossolo, os fatores climáticos intensos e a baixa disponibilidade de nutrientes resultaram em matéria orgânica com menor grau de humificação. Nesses sistemas com mais avançado estádio de intemperismo (Jackson \& Sherman, 1953), especialmente nos Latossolos, os processos de perda tendem a superar os de ganho e a resultante confere um ambiente biológico menos diversificado e mais especializado, com ação regulada pelos ciclos climáticos, da- das as maiores intensidades a que este é submetido (Baldotto et al., 2010). Como exemplos, podem-se citar as menores área superficial específica, geração de cargas elétricas, capacidade de retenção de íons dos coloides e estabilização dos estoques de carbono no solo, indicando desfavorecimento à humificação (Baldotto et al., 2010).

No entanto, aumentos de fertilidade e da concentração de SH foram observados, por Cunha (2005), em alguns solos antropogênicos da região Norte do Brasil. Nesses sistemas, os Latossolos, mesmo sob ambientes favoráveis à decomposição da matéria orgânica do solo (Amazônia), apresentaram horizonte A antrópico (Embrapa, 2013), com elevados estoques de carbono, enriquecido em bases trocáveis, com $\mathrm{pH}$ em torno de 6,5, alta CTC, estrutura bem desenvolvi-

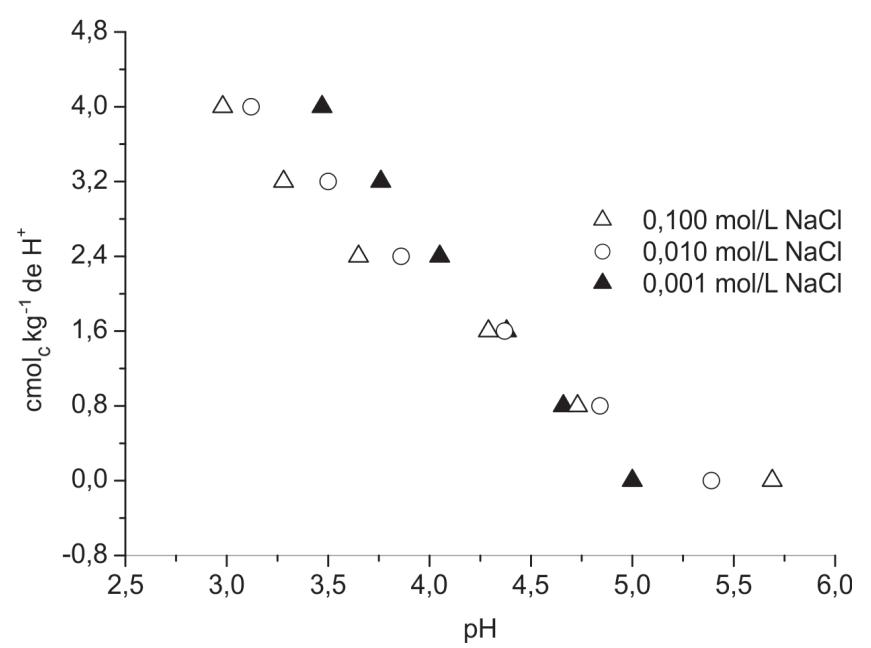

Figura 1. Adsorção de $\mathrm{H}^{+}$e de $\mathrm{OH}^{-}$de acordo com os valores de $\mathrm{pH}$ para as três curvas de titulação de $5 \mathrm{~g}$ de TFSA de um Chernossolo suspenso em $25 \mathrm{~mL}$ das diferentes concentrações de $\mathrm{NaCl}$. O valor de $\mathrm{pH}$ no ponto de cruzamento das curvas é definido como o de efeito salino nulo $(\mathrm{PESN}=4,35)$.

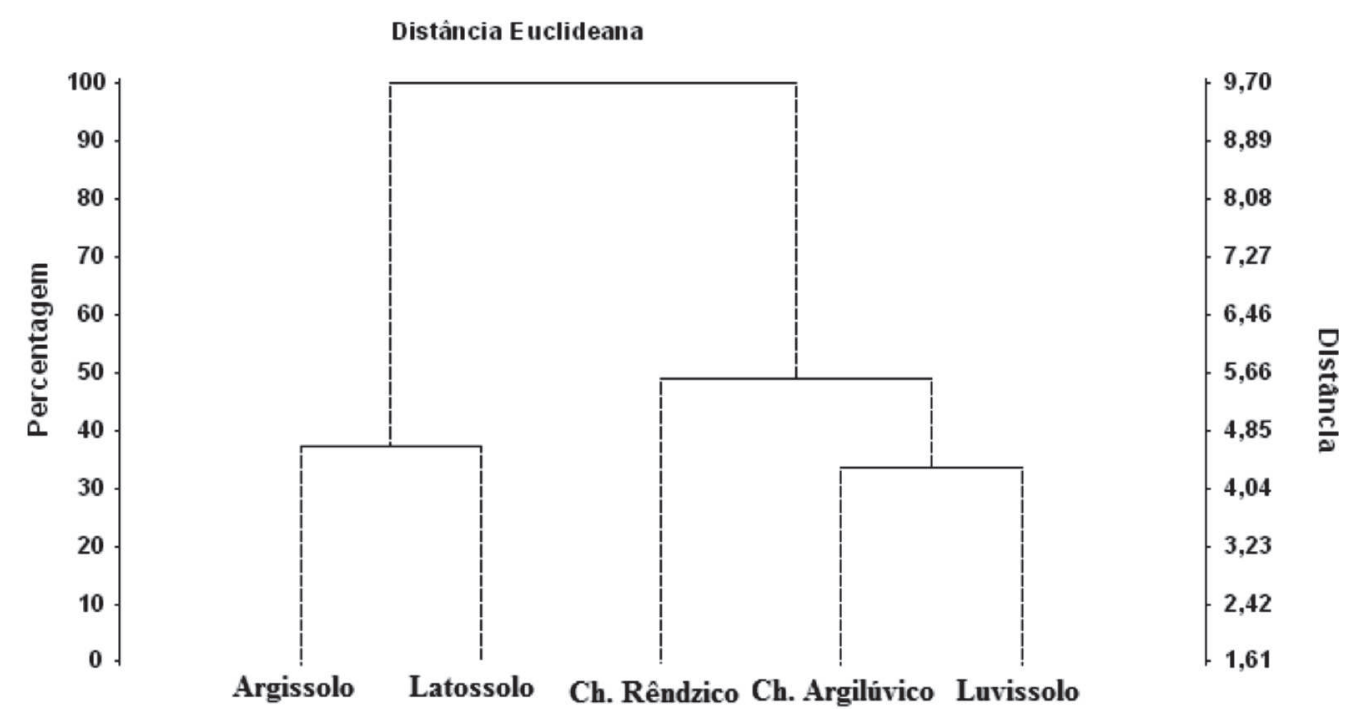

Figura 2. Dendrograma, utilizando a distância Euclidiana, obtido pela análise multivariada de todos os atributos dos solos amostrados apresentados nas Tabelas 1, 2, 3 e 4 . 


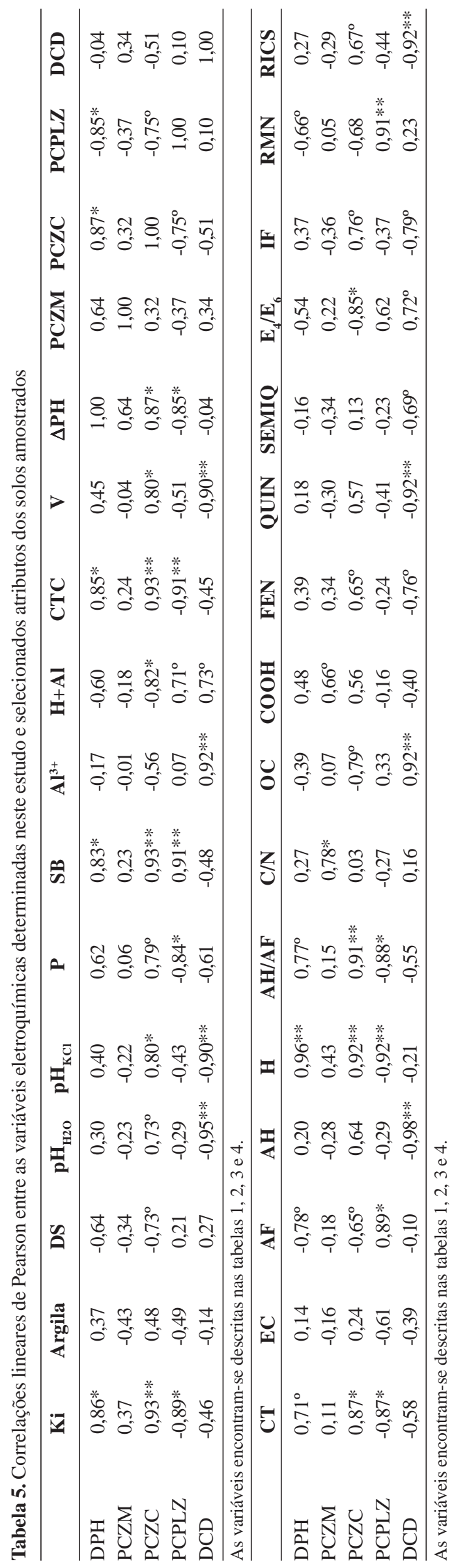

da, possibilitando adequados fluxos da atmosfera e da solução do solo e elevados teores de P, ou seja, alta fertilidade. A natureza da estabilização do húmus nesses sistemas ainda não foi totalmente esclarecida. Existe, contudo, razoável convergência sobre a formação de estruturas condensadas de carbono no húmus, advindas do uso contínuo e conjunto de resíduos minerais e orgânicos (queimados ou não), em áreas de Latossolos. Nesse sentido, os resultados deste trabalho convergem para a necessidade de incrementar a qualidade e a quantidade das formas humificadas de carbono orgânico, nos solos tropicais, pois estes tendem às diminuições de CTC e de disponibilidade de bases e aos aumentos de adsorção de $\mathrm{P}$ e de acidez, fatores antagônicos à fertilidade do solo. Infere-se, portanto, que além das tradicionais práticas de manejo e de conservação do solo, incluindo a integração de florestas à lavoura e à pecuária, o desenvolvimento de novos fertilizantes, combinando fontes orgânicas, minerais e biológicas (Baldotto et al. 2011; 2012a, 2012b), seja a alternativa fundamental para, ciclo após ciclo, inverter o processo de degradação de muitas áreas tropicais, como os solos antropogênicos, os quais são o registro de sucesso tecnológico de adubações orgânico-minerais, em sistemas agroflorestais (Baldotto et al., 2006).

\section{CONCLUSÕES}

Os resultados possibilitaram concluir que os métodos de estimativa do ponto de carga zero (PCZ) apresentam a mesma tendência entre os solos, porém tendo magnitude de resultados variada, evidenciando que a interpretação do valor depende do método usado.

Todos os solos estudados mostram carga líquida negativa, em ambas as camadas amostradas, com menores valores de PCZ e mais negativos $\Psi_{O}$ na camada superficial.

Os solos mais imtemperizados apresentam maiores valores de PCZ (tendência ao caráter mais eletropositivo), valores menos negativos de $\Psi_{O}$ (menor densidade de carga negativa) e menor estoque de carbono.

Os valores estimados para o PCZ e para o $\Psi_{\mathrm{O}}$ relacionam-se, significativamente, com outros atributos físico-químicos fundamentais dos solos e, especialmente, com a eletroquímica da matéria orgânica dos solos, indicando sua forte participação na geração de cargas negativas (capacidade de transferir elétrons).

\section{AGRADECIMENTOS}

Os autores agradecem ao CNPq, à Faperj, à Fapemig e à Funarbe, pelos financiamentos. 


\section{REFERÊNCIAS}

Baldotto MA (2006) Propriedades redox e grupos funcionais de ácidos húmicos. Tese de Doutorado. Universidade Estadual do Norte Fluminense "Darcy Ribeiro", Campos dos Goytacazes. 100p.

Baldotto MA, Canela MC, Canellas LP, Dobbss LB \& Velloso ACX (2010) Redox índex of soil carbon stability. Revista Brasileira de Ciência do Solo, 34:1543-1551.

Baldotto MA, Giro VB, Baldotto LEB, Canellas LP \& Velloso ACX (2011) Initial performance of pineapple and utilization of rock phosphate applied in combination with organic compounds to leaf axils. Revista Ceres, 58:393-401.

Baldotto MA, Baldotto LEB, Gonzaga Junior L, Olivares FL \& Canellas LP (2012a) Initial growth of maize in response to application of rock phosphate,vermicompost and endophytic bacteria. Revista Ceres, 59:262-270.

Baldotto MA, Baldotto LEB, Santana RB \& Marciano CR (2012b) Initial performance of maize in response to application of NPK combined with Herbaspirillum seropedicae. Revista Ceres, 56:841-849.

Charlet L \& Sposito G (1987) Monovalent ion adsorption by an Oxisol. Soil Science Society America Journal, 51:1155-1160

Chaves LHG \& Trajano MDM (1992) Determinação do ponto de carga zero e das cargas elétricas do horizonte Ap de solos do Estado da Paraíba. Revista Brasileira de Ciência do Solo, 16:415-418.

Cruz CD (2006) Análise multivariada e simulação. Viçosa, Editora UFV. $175 \mathrm{p}$.

Cunha TJF (2005) Ácidos húmicos de solos escuros da Amazônia. Tese de doutorado. UFRRJ, Seropédica. 140p.

Embrapa (1980) Centro Nacional de Levantamento e Classificação de Solos. I Reunião de Classificação e Correlação de Solos - BT n ${ }^{\circ} 62$. Rio De Janeiro, EMBRAPA Produção de Informação. 79p.

Embrapa - Empresa Brasileira de Pesquisa Agropecuária (2013) Sistema Brasileiro de Classificação de Solos. $3^{\mathrm{a}}$ ed. Rio de Janeiro, Centro Nacional de Pesquisa em Solos. 353p.

Fontes, MPF, Camargo OA \& Sposito G (2001) Eletroquímica das partículas coloidais e sua relação com a mineralogia de solos altamente intemperizados. Scientia Agrícola, 58:627-646.

Jackson ML \& Sherman GD (1953) Chemical weathering of mineral soils. Advances in Agronomy, 5:219-318.

Leal JR, Amaral Sobrinho NMB, Velloso ACX \& Rossiello ROP (1983) Potencial redox e $\mathrm{pH}$ : variações em um solo tratado com vinhaça. Revista Brasileira de Ciência do Solo, 7:257-261.

Leal JR \& Veloso ACS (1973) Adsorção de fosfato em Latossolo sob vegetação de cerrado. Pesquisa Agropecuária Brasileira, 8:81-88.

Magalhães AF \& Page AL (1984a) Características das cargas elétricas dos solos da Zona da Mata de Pernambuco. I. Determinação das cargas de superfície. Revista Brasileira de Ciência do Solo, 8:173177.

Magalhães AF \& Page AL (1984b) Características das cargas elétricas dos solos da Zona da Mata de Pernambuco. II. Avaliação comparativa entre métodos de determinação. Revista brasileira de Ciência do Solo, 8:178-182.

Manzato HRH, Santos G de A, Velloso ACX \& Pessanha GG (1994) Influência da mistura de material de horizontes de Glei Pouco Húmico no $\mathrm{E}_{\mathrm{H}}$ e no pH. Revista brasileira de Ciência do Solo, 18:21-25.

Oliveira C, Velloso ACX \& Leal JR (1993) Processos redox em Glei Húmico do Estado do Rio de Janeiro: I. Variações eletroquímicas. Revista Brasileira de Ciência do Solo, 17:17-22.

Parks GA \& Bruyn PL (1962) The zero point of charge of oxides. Journal of Physcal Chemistry, 66:967-973.
Parks GA (1965) The isoelectric points of solid oxides, solid hydroxides, and aqueous hydroxo complex systems. Chemical Reviews, 65:177198.

Peixoto RT (1997) Matéria orgânica e a dinâmica das cargas elétricas dos solos: processos e conseqüências. In: $26^{\circ}$ Congresso Brasileiro de Ciência do Solo, Rio de Janeiro. Anais, Sociedade Brasileira de Ciência do Solo/EMBRAPA-CNPS. CD-ROM.

Piccolo A (2001) The supramolecular structure of humic substances. Soil Science, 166:810-832

Raij B Van \& Peech M (1972) Electrochemical properties of some Oxisols and Alfisols of the tropics. Soil Science Society America Journal, 36:587-593.

Raij B van (1973a) Determinação de cargas elétricas em solos. Bragantia, $32: 171-183$

Raij B van (1973b) Determinação do ponto de carga zero em solos. Bragantia, 32:337-347.

Schofield RK (1949) Effect of $\mathrm{pH}$ on electric charges carried by clay particles. Soil Science Society America Journal,1:1-8.

Silva MLN, Curi N, Marques JJGS, Guilherme LRG \& Lima JM (1996) Ponto de efeito salino nulo e suas relações com propriedades mineralógicas e químicas de Latossolos brasileiros. Pesquisa Agropecuária Brasileira, 31:663-671.

Siqueira C, Leal JR, Velloso ACX \& Santos GA (1990a) Eletroquímica de solos tropicais de carga variável: I. Influência da matéria orgânica no tempo de equilíbrio pra a determinação das curvas de titulação potenciométrica. Revista Brasileira de Ciência do Solo, 14:7-11.

Siqueira C, Leal JR, Velloso ACX \& Santos GA(1990b) Eletroquímica de solos tropicais de carga variável: II. Quantificação do efeito da matéria orgânica sobre o ponto de carga zero. Revista Brasileira de Ciência do Solo, 14:13-17.

Siqueira C, Leal JR, Velloso ACX \& Santos GA (1990c) Eletroquímica de solos tropicais de carga variável: III. Erros na avaliação das cargas. Revista Brasileira de Ciência do Solo, 14:19-24.

Sposito G (1981) The operational definition of the zero point of charge in soils. Soil Science Society America Journal, 45:292-297.

Sposito G (1989) Surface reactions in natural aqueous colloidal systems. Chimia, 43:169-176.

Sposito G (1998) On points of zero charge. Envirom. Science \& Technology, 32:2815-2819.

Sposito G (2008) The chemistry of soils. New York, Oxford. 330p.

Stern O (1924) Zur theorie der elektrolytischen doppelschieht. Z. Electrochem, 30:508-516.

Steel RGD \& Torrie JH (1980) Principles and procedures of statistics. $2^{\text {nd }}$. New York, McGraw-Hill. 633p.

Stevenson FJ (1994) Humus chemistry: genesis, composition, reactions. $2^{\text {nd }}$. New York, Wiley. 496p.

Struyk Z \& Sposito G (2001) Redox properties of standard humic acids. Geoderma, 102:329-346.

Stumm W (1992) Chemistry of the solid-water interface. New York, John Wiley \& Sons. 428p.

Sutton R \& Sposito G (2005) Molecular structure in soil humic substances: the new view. Environmental Science Technology, 39:9009-9015.

Uehara G \& Gillman GP (1980a) Charge characteristics of soils with variable and permanent charge minerals: I. Theory. Soil Science Society America Journal, 44:250-255.

Uehara G \& Gillman GP (1980b) Charge characteristics of soils with variable and permanent charge minerals: II. Experimental. Soil Science Society America Journal, 44:252-255. 